\title{
THE VIRASORO GROUP AS A COMPLEX MANIFOLD
}

\author{
LÁSZLÓ LEMPERT
}

\section{Introduction}

The Virasoro group Vir is a central extension of the group Diff, the orientation preserving $C^{\infty}$ diffeomorphisms of the circle $S^{1}=\mathbb{R} / 2 \pi \mathbb{Z}$. As a set, Vir $=$ Diff $\times \mathbb{R}$; the group multiplication is given by

$$
(\varphi, \theta)(\psi, \omega)=(\varphi \circ \psi, \theta+\omega+B(\varphi, \psi)),
$$

where $\varphi, \psi \in$ Diff, $\theta, \omega \in \mathbb{R}$, and $B$ is Bott's cocycle

$$
B(\varphi, \psi)=\int_{0}^{2 \pi} \log (\varphi \circ \psi)^{\prime} d \log \psi^{\prime},
$$

see [2]. Both groups Diff and Vir are in fact Lie groups modeled on Fréchet spaces. They both play an important role in string theory: Diff as the group of reparametrizations of a string, Vir because projective representations of a group lead to central extensions. Locally (on the level of Lie algebras), Vir is the universal central extension of Diff.

In the past ten years many infinite dimensional complex manifolds have been constructed in connection with the representation theory of Vir, see $[1,3,4,9,10,11,12,15,23]$. In particular, if $S^{1}$ is embedded into Diff as the group of translations (= "rotations"), according to Bowick and Rajeev Diff $/ S^{1}$ has a left invariant complex structure, which by Kirillov and Yuriev is biholomorphic to a space of univalent functions. It does not seem to have been noticed, however, that the group Vir itself also carries a natural complex structure.

To formulate our results, let us consider the space $A_{0}(\Delta)$ of smooth, i.e., $C^{\infty}$ complex valued functions on the closure of the unit disc $\Delta \subset \mathbb{C}$ that are holomorphic on $\Delta$ and vanish at 0 . Let $\mathcal{F}$ denote the set of those $f \in A_{0}(\Delta)$ that give a differentiable embedding of $\bar{\Delta}$ into $\mathbb{C}$. Then $A_{0}(\Delta)$ is a Fréchet space over $\mathbb{C}$, and $\mathcal{F}$, as an open subset of $A_{0}(\Delta)$, inherits a complex manifold structure.

Received April 12, 1995.

Supported by the NSF and a grant from the Hungarian Government. 
Theorem 1.1. Vir admits a left invariant complex structure; with this structure it is biholomorphic to $\mathcal{F}$. In fact, there is a family of such complex structures parametrized by non-real complex numbers.

[12] also introduces the complex manifold $\mathcal{F}$ (denoted there by $\mathcal{A}$ ), but the authors very emphatically do not identify it with the Virasoro group. [11] gets closer to Theorem 1.1. The authors construct an action of the Virasoro algebra on $\mathcal{F}$, but they stop short of realizing that this action integrates to a free transitive action of the group itself - which would then identify $\operatorname{Vir}$ and $\mathcal{F}$. As a consequence, the orbit method allows them to construct representations of the Virasoro algebra only. Using our results it will be easy to integrate those representations to group representations. However, we will not pursue this line in the present paper. The integrability of Verma modules has been known for some time now anyway, see $[6,17]$.

As to the group Diff itself, we will prove that it admits an "odd dimensional" counterpart of complex structures:

Theorem 1.2. Diff admits a left invariant Cauchy-Riemann structure(CR for short); with this structure it is isomorphic to a strongly pseudoconvex hypersurface $\mathcal{F}_{1} \subset \mathcal{F}$. In fact,

$$
\mathcal{F}_{1}=\{f \in \mathcal{F}: f(\partial \Delta) \text { has conformal radius } 1\} .
$$

(For definitions, see below.)

The CR structure in Theorem 1.2 is invariant under the right action of $S^{1}$, and this free action is transverse to the CR structure. In such a situation the quotient Diff/ $S^{1}$ inherits a natural complex, even Kähler structure. The complex manifold Diff/ $S^{1}$ thus obtained will be biholomorphic to a corresponding quotient $\mathcal{F}_{1} / S^{1}$, which can be identified with $\mathcal{S}=\{f \in \mathcal{F}$ : $\left.f^{\prime}(0)=1\right\}$. In this way we recover some of the results of $[9,10]$. We do not, however, claim a new proof of the results of Kirillov-Yuriev; indeed, our proof of Theorem 1.2 is but a slight modification of theirs.

\section{Preliminaries, etc.}

We will work with infinite dimensional manifolds as defined in [7] or [16]; all our manifolds will be smooth (meaning $C^{\infty}$ ) and modeled on (real) Fréchet spaces. An almost complex structure on a manifold $\mathcal{M}$ is given by a splitting of the complexified tangent bundle $\mathbb{C} \otimes T \mathcal{M}=T^{1,0} \oplus T^{0,1}$ into two complex subbundles, $\overline{T^{1,0}}=T^{0,1}$. A $C^{1}$ mapping $F$ between two almost 
complex manifolds $\left(\mathcal{M}, T^{1,0} \mathcal{M}\right),\left(\widetilde{\mathcal{M}}, T^{1,0} \widetilde{\mathcal{M}}\right)$ is called holomorphic if its differential maps $T^{1,0} \mathcal{M}$ into $T^{1,0} \widetilde{\mathcal{M}}$. If in addition $F$ has a $C^{1}$ inverse $\widetilde{\mathcal{M}} \rightarrow \mathcal{M}$ then this inverse is also holomorphic, $F$ is called biholomorphic, and $\mathcal{M}, \widetilde{\mathcal{M}}$ are biholomorphic to each other. A complex manifold is a manifold $\mathcal{M}$ endowed with an integrable almost complex structure $T^{1,0}$ i. e. , one such that $\left[T^{1,0}, T^{1,0}\right] \subset T^{1,0}$ (meaning the Lie bracket of two sections of $T^{1,0}$ is again a section of $\left.T^{1,0}\right)$. For example, if $\mathcal{M}$ is a complex Fréchet space, multiplication by $i$ defines an endomorphism $J: \mathcal{M} \rightarrow \mathcal{M}$ with $J^{2}=-$ id. Also, the (real!) tangent spaces $T_{f} \mathcal{M}(f \in \mathcal{M})$ can be identified with $\mathcal{M}$, and $J$ gives rise to endomorphisms $J_{f}: T_{f} \mathcal{M} \rightarrow T_{f} \mathcal{M}$. Putting $T^{1,0}=\left\{v-i J_{f} v \in \mathbb{C} \otimes T_{f} \mathcal{M}: f \in \mathcal{M}, v \in T_{f} \mathcal{M}\right\}$ we obtain the usual complex manifold structure on $\mathcal{M}$.

The Newlander-Nirenberg theorem asserts that any finite dimensional complex manifold as defined above is locally biholomorphic to a complex vector space (see [18]). For infinite dimensional manifolds this is not true; some interesting complex manifolds not locally biholomorphic to complex vector spaces can be found in $[13,14]$.

If $\mathcal{G}$ is a possibly infinite dimensional Lie group with Lie algebra $\mathfrak{g}$, a left invariant almost complex structure on $\mathcal{G}$ is determined by a splitting $\mathbb{C} \otimes$ $T_{e} \mathcal{G}=\mathbb{C} \otimes \mathfrak{g}=\mathfrak{g}^{1,0} \oplus \mathfrak{g}^{0,1}$, with $\mathfrak{g}^{1,0}$ and $\mathfrak{g}^{0,1}=\overline{\mathfrak{g}^{1,0}}$ complex subspaces. The almost complex structure is integrable if $\mathfrak{g}^{1,0}$ is a subalgebra. This complex structure is invariant under right translation by a $g \in \mathcal{G}$ if $\mathrm{A} d_{g} \mathfrak{g}^{1,0}=\mathfrak{g}^{1,0}$.

Similarly, an almost CR structure on a manifold $\mathcal{N}$ is a splitting $H^{1,0} \oplus$ $H^{0,1}$ of a corank 1 subbundle of $\mathbb{C} \otimes T \mathcal{N}$, with $\overline{H^{1,0}}=H^{0,1}$, and it is called an integrable almost $\mathrm{CR}$ structure, or just CR structure, if $\left[H^{1,0}, H^{1,0}\right] \subset$ $H^{1,0}$. For example, a smooth real hypersurface $\mathcal{N}$ in a complex manifold $\left(\mathcal{M}, T^{1,0}\right)$ inherits a CR structure $H^{1,0}=\left.T^{1,0}\right|_{\mathcal{N}} \cap \mathbb{C} \otimes T \mathcal{N}$. A CR manifold $\left(\mathcal{N}, H^{1,0}\right)$ is strongly pseudoconvex if for any local section $X$ of $H^{1,0} X(p) \neq$ 0 implies $[X, \bar{X}](p) \notin H_{p}^{1,0} \oplus H_{p}^{0,1}$. An isomorphism between CR manifolds $\left(\mathcal{N}, H^{1,0} \mathcal{N}\right),\left(\widetilde{\mathcal{N}} ; H^{1,0} \tilde{\mathcal{N}}\right)$ is a $C^{1}$ diffeomorphism between $\mathcal{N}$ and $\widetilde{\mathcal{N}}$ whose differential carries $H^{1,0} \mathcal{N}$ to $H^{1,0} \widetilde{\mathcal{N}}$. A $C^{1}$ mapping $F$ between a $\mathrm{CR}$ manifold $\left(\mathcal{N}, H^{1,0} \mathcal{N}\right)$ and a complex manifold $\left(\mathcal{M}, T^{1,0} \mathcal{M}\right)$ is called CR if its differential maps $H^{1,0} \mathcal{N}$ into $T^{1,0} \mathcal{M}$. Finally, on a Lie group $\mathcal{G}$ with Lie algebra $\mathfrak{g}$ a left invariant $\mathrm{CR}$ structure is determined by a splitting $\mathfrak{h}^{1,0} \oplus \mathfrak{h}^{0,1}$ of a codimension 1 subspace of $\mathbb{C} \otimes \mathfrak{g}$, with $\mathfrak{h}^{1,0}$ and $\mathfrak{h}^{0,1}=\overline{\mathfrak{h}^{1,0}}$ subalgebras. This structure is strongly pseudoconvex if $[v, \bar{v}] \notin \mathfrak{h}^{1,0} \oplus \mathfrak{h}^{0,1}$ holds for any nonzero $v \in \mathfrak{h}^{1,0}$.

A few words about the formalism of complex analysis on manifolds. Dif- 
ferential forms and exterior differentiation $d$ are defined on smooth manifolds $\mathcal{M}$ as in the finite dimensional situation $([7,16,20])$. On a complex manifold $\left(\mathcal{M}, T^{1,0}\right)$

$$
\bigwedge^{k} \mathbb{C} \otimes T \mathcal{M}=\bigoplus_{i+j=k} \bigwedge^{i} T^{1,0} \otimes \bigwedge^{j} T^{0,1},
$$

and a complex valued $k$-form is called a $(p, q)$-form $(p+q=k)$ if it vanishes on all components $\bigwedge^{i} T^{1,0} \otimes \bigwedge^{j} T^{0,1}$ except possibly when $i=p, j=q$. If $\alpha$ is a smooth $(p, q)$-form, $d \alpha=\beta^{\prime}+\beta^{\prime \prime}$ with $\beta^{\prime}$, resp., $\beta^{\prime \prime}$, a $(p+1, q)$, resp., $(p, q+1)$-form. The operators $\partial, \bar{\partial}$ are defined by $\partial \alpha=\beta^{\prime}, \bar{\partial} \alpha=$ $\beta^{\prime \prime}$. While basic real analysis on a smooth infinite dimensional manifold is rather similar to the finite dimensional case - in particular, on a contractible manifold $d$-closed forms are exact - the infinite dimensional theory of the Cauchy-Riemann operator $\bar{\partial}$ is a largely uncharted territory. Of the few results on solving the Cauchy-Riemann equations in infinite dimensions, we will find those in [21] very useful for the purpose of this paper.

Just like $d, \partial$ and $\bar{\partial}$ can be defined more generally for smooth (or $C^{1}$ ) mappings $F$ between almost complex manifolds $\left(\mathcal{M}, T^{1,0} \mathcal{M}\right),\left(\widetilde{\mathcal{M}}, T^{1,0} \widetilde{M}\right)$. Indeed, $d F$ maps $\mathbb{C} \otimes T \mathcal{M}$ to $\mathbb{C} \otimes T \widetilde{\mathcal{M}}$. Restricting to $T^{1,0} \mathcal{M}$, resp., $T^{0,1} \mathcal{M}$, and composing with the projection on $T^{1,0} \widetilde{\mathcal{M}}$ we obtain the complex differentials

$$
\partial F: T^{1,0} \mathcal{M} \rightarrow T^{1,0} \widetilde{\mathcal{M}}, \quad \bar{\partial} F: T^{0,1} \mathcal{M} \rightarrow T^{1,0} \widetilde{\mathcal{M}}
$$

The mapping is holomorphic if $\bar{\partial} F=0$. Later we will need a "Leibniz rule" in this context. In addition to $\mathcal{M}$, suppose a group $\mathcal{G}$ is also given, with left invariant almost complex structure $T^{1,0} \mathcal{G}$, and let $\alpha, \beta$ be smooth maps from $\mathcal{M}$ to $\mathcal{G}$. Then

$$
d(\alpha \beta)=\alpha d \beta+(d \alpha) \beta,
$$

where on the right the first term means $d \beta$ followed by left translation by $\alpha$, and the second means $d \alpha$ followed by right translation by $\beta$ (translations acting on $T \mathcal{G}$ ). In general, there is no corresponding formula for $\bar{\partial}$. Suppose, however, that $\mathcal{C} \subset \mathcal{G}$ is the subgroup consisting of elements $g$ such that $T^{1,0} \mathcal{G}$ is invariant under right translation by $g$, and $\beta$ maps into $\mathcal{C}$. Then (2.1) implies

$$
\bar{\partial}(\alpha \beta)=\alpha \bar{\partial} \beta+(\bar{\partial} \alpha) \beta
$$




\section{The CR structure on Diff}

The Lie algebra diff $=T_{\text {id }}$ Diff can be identified with vect, the space of smooth vector fields on $S^{1}$; if Lie bracket on diff is defined using commutators of left invariant vector fields on Diff, this Lie bracket turns out to be the negative of the bracket of vector fields on $S^{1}$ (cf. [16]). Below we will use [ , ] to denote Lie bracket on diff, thus

$$
\left[e^{i n t} \frac{d}{d t}, e^{i k t} \frac{d}{d t}\right]=i(n-k) e^{i(n+k) t} \frac{d}{d t}
$$

holds on $\mathbb{C} \otimes$ diff $=\mathbb{C} \otimes$ vect. $\left(t\right.$ denotes the coordinate on $S^{1}=\mathbb{R} / 2 \pi \mathbb{Z}$.) Define

$$
\mathfrak{h}^{1,0}=\left\{\sum_{n=1}^{\infty} a_{n} e^{i n t} \frac{d}{d t} \in \mathbb{C} \otimes \operatorname{vect}\right\},
$$

a subalgebra. $\mathfrak{h}^{1,0} \oplus \overline{\mathfrak{h}^{1,0}}$ is of codimension one in $\mathbb{C} \otimes$ vect, whence we obtain a left invariant CR structure $H^{1,0}$ on Diff. In addition,

$$
\left[\sum_{1}^{\infty} a_{n} e^{i n t} \frac{d}{d t}, \sum_{1}^{\infty} \bar{a}_{n} e^{-i n t} \frac{d}{d t}\right]=i \sum_{k, n=1}^{\infty}(k+n) a_{n} \bar{a}_{k} e^{i(n-k) t} \frac{d}{d t}
$$

which is not in $\mathfrak{h}^{1,0} \oplus \overline{\mathfrak{h}^{1,0}}$ unless all $a_{n}=0$. Thus (Diff, $H^{1,0}$ ) is strongly pseudoconvex. To finish off the proof of Theorem 1.2 we need to embed Diff into $\mathcal{F}$.

First a word about conformal radius. Given a Jordan curve $\Gamma \subset \mathbb{C}$, there is a univalent function $g(\zeta)=\beta \zeta+\beta_{0}+\beta_{-1} \zeta^{-1}+\ldots$ that maps $\mathbb{C} \backslash \Delta$ on the exterior of $\Gamma$. This $g$ is unique up to rotation of $\zeta$, and the number $|\beta|>0$ is called the conformal radius of $\Gamma$. As $f$ ranges over $\mathcal{F}$, the conformal radius of $f(\partial \Delta)$, denoted $\beta(f)$, depends smoothly on $f$; furthermore $\beta(\gamma f)=|\gamma| \beta(f)$ for $\gamma \in \mathbb{C} \backslash\{0\}=\mathbb{C}^{*}$. This implies that $\mathcal{F}_{1}$ of Theorem 1.2 is a smooth hypersurface in $\mathcal{F}$. Indeed, the mapping

$$
\mathcal{S} \times \mathbb{C}^{*} \ni(h, \alpha) \mapsto \alpha h / \beta(h) \in \mathcal{F}
$$

is a diffeomorphism, and $\mathcal{F}_{1}$ is the image of $\mathcal{S} \times \partial \Delta$.

Next we define a mapping $p: \mathcal{F}_{1} \rightarrow$ Diff as follows. Given $f \in \mathcal{F}_{1}$, let $g(\zeta)=\zeta+\beta_{0}+\beta_{-1} \zeta^{-1}+\ldots$ be the univalent function that maps $\mathbb{C} \backslash \Delta$ on the exterior of $f(\partial \Delta)$. As $g$ extends to a diffeomorphism between the 
boundaries, we can form $P(f)=f^{-1} \circ g=\Phi \in$ Diff $\partial \Delta$ and $p(f)=\varphi \in$ Diff defined by

$$
\Phi\left(e^{i t}\right)=e^{i \varphi(t)} .
$$

Since $g$ depends smoothly on $f(\partial \Delta)$, we see that $P$ and $p$ are smooth on $\mathcal{F}_{1}$. To verify $p$ has an inverse it must be shown that any $\Phi \in \operatorname{Diff} \partial \Delta$ can be uniquely represented as $f^{-1} \circ g$ with $f, g$ as above, which is a theorem of Pfluger, see [19]. (Pfluger treats homeomorphisms $\Phi$ of $\partial \Delta$ with very little regularity, and gets $f, g$ that have correspondingly little boundary regularity, but the proof yields $f \in \mathcal{F}_{1}$ when $\Phi$ is a diffeomorphism. Also $f$ depends smoothly on $\Phi$.) Hence $p$ is a diffeomorphism between $\mathcal{F}_{1}$ and Diff.

To verify $p$ is a CR isomorphism, we introduce a trivialization $T$ Diff $\simeq$ Diff $\times$ diff as follows. A smooth curve $\varphi_{\epsilon}$ in Diff determines a tangent vector $w$ in $\varphi_{0}=\varphi \in$ Diff. The derivative $\partial \varphi_{\epsilon} /\left.\partial \epsilon\right|_{\epsilon=0}=\dot{\varphi}$ is a smooth real function on $S^{1}$. In the sequel $w$ will be represented by $(\varphi ; \dot{\varphi})$ or $(\varphi ; \dot{\varphi} d / d t) \in$ Diff $\times$ diff. It is immediate to check that the left translate of $v \in T_{\mathrm{id}}$ Diff to $\varphi \in$ Diff is

$$
L_{\varphi} v=\left(\varphi ; \varphi^{\prime} v\right)
$$

and the same formula holds for complexified tangent vectors. Here $\varphi^{\prime}=$ $d \varphi / d t$. On the other hand, if $h$ is a smooth function on $\partial \Delta, h^{\prime}(\zeta)$ will denote $\lim (h(\omega)-h(\zeta)) /(\omega-\zeta)$ as $\partial \Delta \ni \omega \rightarrow \zeta$.

To understand the differentials of $p, P$, suppose $f_{\epsilon}$ is a smooth curve in $\mathcal{F}_{1}$ through $f=f_{0}$, and let $g_{\epsilon}(\zeta)=\zeta+\beta_{0}(\epsilon)+\ldots$ be the univalent map of $\mathbb{C} \backslash \Delta$ on the exterior of $f_{\epsilon}(\partial \Delta)$. With $\Phi_{\epsilon}=P\left(f_{\epsilon}\right)$ we have

$$
g_{\epsilon}=f_{\epsilon} \circ \Phi_{\epsilon} \quad \text { on } \partial \Delta .
$$

Indicating $\partial /\left.\partial \epsilon\right|_{\epsilon=0}$ by a dot, and $g_{0}, \Phi_{0}$ by $g, \Phi,(3.3)$ implies

$$
\dot{g}=\dot{f} \circ \Phi+\left(f^{\prime} \circ \Phi\right) \dot{\Phi}
$$

Here $\dot{f} \in A_{0}(\Delta) \simeq T_{f} \mathcal{F}$ with the usual identification corresponds to the tangent vector $V$ determined by the curve $f_{\epsilon}$.

Now suppose this tangent vector is the real part of a vector $W \in H_{f}^{1,0} \mathcal{F}_{1}$. Then $W=V-i J_{f} V$ with notation as in section 2 , and $J_{f} V$ is the tangent 
vector of a curve $f_{\epsilon}^{*}$ with $\dot{f}^{*}=i \dot{f}$. With corresponding $g_{\epsilon}^{*}, \Phi_{\epsilon}^{*}$, etc., we have as in (3.4)

$$
\dot{g}^{*}=\dot{f}^{*} \circ \Phi+\left(f^{\prime} \circ \Phi\right) \dot{\Phi}^{*} .
$$

Multiply by $i$ and add to (3.4):

$$
\dot{g}+i \dot{g}^{*}=\left(f^{\prime} \circ \Phi\right)\left(\dot{\Phi}+i \dot{\Phi}^{*}\right) .
$$

On the other hand, in (3.3) put $\epsilon=0$ and differentiate to get $g^{\prime}=\left(f^{\prime} \circ \Phi\right) \Phi^{\prime}$, so that

$$
\left(\dot{g}+i \dot{g}^{*}\right) / g^{\prime}=\left(\dot{\Phi}+i \dot{\Phi}^{*}\right) / \Phi^{\prime} .
$$

Thus the right hand side extends to a holomorphic function on $\mathbb{C} \cup\{\infty\} \backslash \bar{\Delta}$.

Put $\varphi_{\epsilon}=p\left(f_{\epsilon}\right), \varphi_{\epsilon}^{*}=p\left(f_{\epsilon}^{*}\right)$, etc. Attaching $\epsilon$ subscripts to (3.1) and differentiating we find

$$
\frac{\dot{\Phi}\left(e^{i t}\right)+i \dot{\Phi}^{*}\left(e^{i t}\right)}{\Phi^{\prime}\left(e^{i t}\right)}=i e^{i t} \frac{\dot{\varphi}(t)+i \dot{\varphi}^{*}(t)}{\varphi^{\prime}(t)},
$$

whence the Fourier series of $\left(\dot{\varphi}+i \dot{\varphi}^{*}\right) / \varphi^{\prime}$ contains only negative frequencies and so $\left(\dot{\varphi}-i \dot{\varphi}^{*}\right) / \varphi^{\prime}=\sum_{1}^{\infty} a_{n} e^{i n t}$. Note the differential of $p$ maps $W \in H_{f}^{1,0} \mathcal{F}_{1}$ to $w=\left(\varphi ; \dot{\varphi}-i \dot{\varphi}^{*}\right) \in \mathbb{C} \otimes T_{\varphi}$ Diff, and this vector is in $H_{\varphi}^{1,0}$ Diff, since it is the left translate of $v=\left(\mathrm{id} ; \sum_{1}^{\infty} a_{n} e^{i n t}\right) \in H_{\mathrm{id}}^{1,0}$ Diff in view of (3.2). Thus any $W \in H^{1,0} \mathcal{F}_{1}$ gets mapped into $H^{1,0}$ Diff, that is, $p$ is a CR isomorphism.

It is worth observing that $H^{1,0}$ is not only left invariant, but also right invariant under $S^{1} \subset$ Diff. It follows that Diff $/ S^{1}$ is a complex manifold, homogeneous under the left action of Diff. Denote the image of id $\in$ Diff in Diff $/ S^{1}$ by [id]. If elements of $T_{\text {[id] }}\left(\right.$ Diff $\left./ S^{1}\right)$ are identified with functions in $C^{\infty}\left(S^{1}\right)$ with zero mean value, then

$$
T_{[\mathrm{id}]}^{1,0}\left(\mathrm{Diff} / S^{1}\right)=\left\{\sum_{1}^{\infty} a_{n} e^{i n t} \in \mathbb{C} \otimes T_{[\mathrm{id}]}\left(\mathrm{Diff} / S^{1}\right)\right\}
$$

This is of course the same complex structure as in $[3,4,9,10]$.

In the remaining part of this section we will construct a family of CR mappings of Diff into an infinite dimensional Grassmannian (which factor through a holomorphic embedding of Diff $/ S^{1}$ into the Grassmannian). In [11] Kirillov and Yuriev construct an analogous embedding of Diff/ $S^{1}$ into a different Grassmannian; below we will try to be more explicit than [11]. 
In [15] Mickelsson constructs, with a minor difference, the same mapping Diff $\rightarrow$ Gr as we will do when the parameter $\lambda$ to be introduced equals one half; he does not, however investigate holomorphicity of this mapping. Actually, in his case the induced embedding Diff $/ S^{1} \rightarrow \mathrm{Gr}$ is antiholomorphic.

For $r \in \mathbb{Z}$ let $H^{r}$ denote the Sobolev space of order $r$ on $S^{1}, H^{0}=H=$ $L^{2}\left(S^{1}\right)$. Introduce a polarization $H^{r}=H_{+}^{r} \oplus H_{-}^{r}$, where $H_{+}^{r}$ consists of those $h \in H^{r}$ whose Fourier series contain only negative frequencies, and $H_{-}^{r}$ is the orthogonal complement of $H_{+}^{r}$. The Grassmannian Gr is the complex Hilbert manifold of subspaces $Z \subset H$ such that the projection of $Z$ on $H_{+}=H_{+}^{0}$ is Fredholm, and its projection on $H_{-}=H_{-}^{0}$ is HilbertSchmidt (see [20]; a minor difference from [20] is that the roles of $H_{+}, H_{-}$ have been reversed, but this is precisely the reason why $\mathrm{CR}$ mappings will be obtained.) For $Z$ near the base point $H_{+}$the projection $Z \rightarrow H_{+}$is invertible, and composing its inverse with the projection $Z \rightarrow H_{-}$we obtain a Hilbert-Schmidt operator $H_{+} \rightarrow H_{-}$that serves as local coordinate of $Z$. Below we will denote the space of continuous operators between Hilbert spaces $A, B$ by $L(A, B)$, and the space of Hilbert-Schmidt operators by $L^{2}(A, B)$. Further we will denote by $\mathrm{Gr}^{\star}$ the open set of $Z \in \mathrm{Gr}$ for which the projection $Z \rightarrow H_{+}$is invertible; the local coordinate constructed above sets up a biholomorphism between $\mathrm{Gr}^{\star}$ and $L^{2}\left(H_{+}, H_{-}\right)$.

Fix a complex number $\lambda$ with $\operatorname{Re} \lambda=\frac{1}{2}$. With any $\varphi \in$ Diff we can associate a unitary operator $U_{\varphi}$ on $H$, given by

$$
U_{\varphi^{-1}} h=\left(\varphi^{\prime}\right)^{\lambda} h \circ \varphi
$$

This representation of Diff on $H$ is not continuous (in operator norm). However, formula (3.6) makes sense on $H^{r}$ as well, and the mapping

$$
\text { Diff } \ni \varphi \mapsto U_{\varphi^{-1}} \in L\left(H^{r}, H^{r-2}\right)
$$

is not only continuous, but continuously differentiable; its derivative along a vector $(\varphi, \dot{\varphi}) \in T$ Diff is

$$
H^{r} \ni h \mapsto \lambda\left(\varphi^{\prime}\right)^{\lambda-1} \dot{\varphi}^{\prime} h \circ \varphi+\left(\varphi^{\prime}\right)^{\lambda} \dot{\varphi} h^{\prime} \circ \varphi \in H^{r-2} .
$$

Theorem 3.1. The formula

$$
\varphi \mapsto U_{\varphi}\left(H_{+}\right)
$$


defines a CR mapping from Diff to $G r^{\star} \subset G r$.

Proof. [20, Section 6.8] implies that $U_{\varphi}\left(H_{+}\right)$is in Gr when $\lambda=\frac{1}{2}$. Our approach to the more precise Theorem 3.1 will be similar to [20]. Introduce projections $\pi_{ \pm}: H^{r} \rightarrow H_{ \pm}^{r}$ and the unitary operator $j=\pi_{+}-\pi_{-}$. One can compute the kernel $K(s, t)$ of the commutator $\left[\pi_{+}, U_{\varphi}\right]$ :

$$
2 \pi K(s, t)=\frac{\varphi^{\prime}(s)^{1-\lambda}}{e^{i(\varphi(s)-t)}-1}-\frac{\psi^{\prime}(t)^{\lambda}}{e^{i(s-\psi(t))}-1},
$$

where $\psi=\varphi^{-1}$. (Similar computations are done in [20, section 6.8].) A change of variables $u=\psi(t)$ will convince us that $K$ is a smooth function that smoothly depends on $\varphi$. It follows that $\left[\pi_{+}, U_{\varphi}\right]$ is Hilbert-Schmidt from $H^{r}$ to $H^{r+\rho}$ for any $r, \rho \in \mathbb{Z}$, and the same holds for $\left[\pi_{-}, U_{\varphi}\right]$; moreover, the mappings

$$
\text { Diff } \ni \varphi \mapsto\left[\pi_{ \pm}, U_{\varphi}\right] \in L^{2}\left(H^{r}, H^{r+\rho}\right)
$$

are smooth. Upon composing with $\pi_{\mp}$ from the right it follows that

$$
\text { Diff } \ni \varphi \mapsto \pi_{ \pm} U_{\varphi} \pi_{\mp} \in L^{2}\left(H^{r}, H^{r+\rho}\right)
$$

are also smooth.

Proposition 3.2. The (operator) norm of the operators $\pi_{+} U_{\varphi} \pi_{-}$and $\pi_{-} U_{\varphi} \pi_{+}$on $H$ is less than 1 .

Accepting this for the moment it follows that the norm of the sum of these two operators is also less than 1 , whence

$$
\pi_{+} U_{\varphi} \pi_{+}+\pi_{-} U_{\varphi} \pi_{-}=j U_{\varphi} j\left\{I+j U_{\varphi^{-1}} j\left(\pi_{+} U_{\varphi} \pi_{-}+\pi_{-} U_{\varphi} \pi_{+}\right)\right\}
$$

is invertible on $H$. Also the inverse, to be denoted $T_{\varphi}$, when regarded as an element of $L\left(H, H^{-2}\right)$, is a $C^{1}$ function of $\varphi$. Restricting to $H_{+}$we see that $\left.\pi_{+} T_{\varphi}\right|_{H_{+}}$is the inverse of $\left.\pi_{+} U_{\varphi}\right|_{H_{+}}$, which implies that $Z=U_{\varphi}\left(H_{+}\right) \in$ $\mathrm{Gr}^{\star}$, and the local coordinate of $Z$ discussed above is the Hilbert-Schmidt operator

$$
\Xi(\varphi)=\left.\pi_{-} U_{\varphi} \pi_{+} T_{\varphi}\right|_{H_{+}} \in L^{2}\left(H_{+}, H_{-}\right) .
$$

By virtue of (3.10) and the smoothness of $T_{\varphi}$, the left hand side of (3.11) is a $C^{1}$ function of $\varphi$. Hence (3.9) defines a $C^{1}$ mapping. (Even better, a 
$C^{\infty}$ mapping. This follows if we note that $U_{\varphi} \in L\left(H^{r}, H^{r-\rho-1}\right)$ is a $C^{\rho}$ function of $\varphi$ for $\rho=1,2, \ldots$ )

It remains to verify that (3.9) is CR. In view of the fact that $U_{\varphi \psi}\left(H_{+}\right)=$ $U_{\varphi}\left(U_{\psi}\left(H_{+}\right)\right)$and the invariance of the CR structure of Diff, it suffices to check this in the point id $\in$ Diff. Using (3.8) we compute the derivative of $\Xi$ in (3.11) in the direction $v=V d / d t \in$ diff: it is the operator

$$
H_{+} \ni h \mapsto-\pi_{-}\left(\lambda V^{\prime} h+V h^{\prime}\right) \in H_{-} .
$$

The same holds for $v \in \mathbb{C} \otimes$ diff. In particular, if $v \in \mathfrak{h}^{0,1}$, then $V$ contains only negative frequency terms. Hence, this operator is zero and (3.9) is indeed CR.

Proof of Proposition 3.2. It will suffice to prove that $D=\pi_{+} U_{\varphi^{-1}} \pi_{-}+$ $\pi_{-} U_{\varphi} \pi_{+}$has norm less than 1 on $H$. It is obvious that this norm is at most $1 ; D$ being compact and self-adjoint we would therefore be done if we could show that \pm 1 is not an eigenvalue of $D$. Now $\|D h\|<\|h\|$ unless $U_{\varphi^{-1}} \pi_{-} h \in H_{+}$and $U_{\varphi} \pi_{+} h \in H_{-}$, so that supposing $D$ had an eigenvector $h$ with eigenvalue \pm 1 we would have $U_{\varphi^{-1}} \pi_{-} h \in H_{+}, U_{\varphi} \pi_{+} h \in$ $H_{-}$. Assume, e.g., that $h_{-}=\pi_{-} h \neq 0$, put

$$
h_{+}=U_{\varphi^{-1}} h_{-}
$$

and define $k_{ \pm} \in L^{2}(\partial \Delta)$ by $k_{ \pm}\left(e^{i t}\right)=h_{ \pm}(t)$. Furthermore, define $\Phi \in$ Diff $\partial \Delta$ by (3.1) and choose $f \in \mathcal{F}_{1}$ and $g$ univalent on $\mathbb{C} \backslash \Delta$ such that $\Phi=f^{-1} \circ g$ on $\partial \Delta$. Then (3.13) implies

$$
k_{+}(\zeta)=(\zeta \Phi(\zeta))^{\lambda} \Phi^{\prime}(\zeta)^{\lambda} k_{-}(\Phi(\zeta)), \quad \zeta \in \partial \Delta
$$

or, putting $\omega=g(\zeta)$, expressing $\Phi$ through $f, g$, and collecting like terms

$$
k_{+}\left(g^{-1}(\omega)\right)\left\{\frac{\left(g^{-1}\right)^{\prime}(\omega)}{g^{-1}(\omega) / \omega}\right\}^{\lambda}=k_{-}\left(f^{-1}(\omega)\right)\left\{\frac{\left(f^{-1}\right)^{\prime}(\omega)}{f^{-1}(\omega) / \omega}\right\}^{\lambda},
$$

for $\omega \in f(\partial \Delta)$. The left hand side extends holomorphically to the exterior of $f(\partial \Delta)$, including $\infty$, and in $\infty$ this extension vanishes. The right hand side extends holomorphically to the interior of $f(\partial \Delta)$. This implies that both sides vanish identically, as do $k_{-}$and $h_{-}$. This is a contradiction, which proves Proposition 3.2. 


\section{The Virasoro group}

The Lie algebra vir of the Virasoro group can be identified with $\operatorname{diff} \times \mathbb{R}$; the Lie bracket of $(v, \xi),(w, \eta) \in \operatorname{diff} \times \mathbb{R}$ is given by

$$
[(v, \xi),(w, \eta)]=([v, w], c(v, w)) .
$$

The bracket on the right comes from diff, and $c$ is the Gelfand-Fuks cocycle $([5,22])$

$$
c\left(V \frac{d}{d t}, W \frac{d}{d t}\right)=\int_{0}^{2 \pi} V^{\prime} d W^{\prime}-W^{\prime} d V^{\prime} .
$$

The same formulae yield the bracket on $\mathbb{C} \otimes$ vir but with complex $\xi, \eta$ and complexified vector fields $v, w$.

Fix a $\tau \in \mathbb{C} \backslash \mathbb{R}$, and define subalgebras

$$
\operatorname{vir}^{1,0}=\left\{\left(\sum_{0}^{\infty} a_{n} e^{i n t} \frac{d}{d t}, \tau a_{0}\right) \in \mathbb{C} \otimes \operatorname{vir}\right\}
$$

and $\operatorname{vir}^{0,1}=\overline{\operatorname{vir}^{1,0}}$. Since $\mathbb{C} \otimes$ vir $=\operatorname{vir}^{1,0} \oplus \operatorname{vir}^{0,1}$, we obtain a left invariant complex structure $T^{1,0}$ on Vir. Composition of the projections Vir $\rightarrow$ Diff and Diff $\rightarrow$ Diff $/ S^{1}$ exhibits Vir as a bundle $\pi:$ Vir $\rightarrow$ Diff $/ S^{1}$. A comparison between (3.5) and (4.1) shows that $\pi$ is holomorphic in the point id $\in$ Vir; as $\pi$ is equivariant under the left action of Vir, this implies that it is holomorphic everywhere. More is true:

Proposition 4.1. $\pi:$ Vir $\rightarrow$ Diff $/ S^{1}$ is a holomorphic principal $\mathbb{C}^{*}$ bundle.

Proof. The Riemann surface $\pi^{-1}([\mathrm{id}])=C$ is seen to be a commutative subgroup of Vir (in fact it is a Cartan subgroup). Let $\mathfrak{c}$ be its Lie algebra. The complex structure of $C$ being left, hence, right invariant, $C$ is a complex Lie group. By inspecting its topological type we find it is isomorphic to $\mathbb{C}^{*}$, the multiplicative group of nonzero complex numbers. Next we claim that the map

$$
\operatorname{Vir} \times C \ni(g, h) \mapsto g h \in \mathrm{Vir}
$$

is holomorphic. Indeed, one checks $\left[\operatorname{vir}^{1,0}, \mathfrak{c}\right] \subset \operatorname{vir}^{1,0}$, whence $\operatorname{Ad}_{h} \operatorname{vir}^{1,0}=$ $\operatorname{vir}^{1,0}$ for $h \in C$. It follows that for fixed $h$ the map (4.2) is holomorphic. Since it is also holomorphic for fixed $g$ by the left invariance of the complex structure on Vir, (4.2) is holomorphic. 
Finally, the $\mathbb{C}^{*} \cong C$ action on Vir given by (4.2) is free, and the projection $\operatorname{Vir} \rightarrow \operatorname{Vir} / C$ is just $\pi$ (after the obvious identification $\operatorname{Vir} / C \approx$ Diff $\left./ S^{1}\right)$. This then proves the Proposition.

Observe that the space $\mathcal{F}$ of univalent functions also admits a $\mathbb{C}^{*}$ action

$$
\mathcal{F} \times \mathbb{C}^{*} \ni(f, z) \mapsto z f \in \mathcal{F}
$$

which exhibits $\mathcal{F}$ as a holomorphic principal $\mathbb{C}^{*}$ bundle. The space of normalized univalent functions $\mathcal{S}$ being a global slice, $\mathcal{F}$ is in fact a holomorphically trivial principal $\mathbb{C}^{*}$ bundle over $\mathcal{S}$. According to [9], Diff/ $S^{1}$ and $\mathcal{S}$ are biholomorphic; therefore, Theorem 1.1 will follow from

Theorem 4.2. $\pi:$ Vir $\rightarrow$ Diff $/ S^{1}$ is a holomorphically trivial principal $\mathbb{C}^{*}$ bundle.

To prove this, a holomorphic section must be exhibited. An obvious smooth section is obtained by associating with the class $[\varphi] \in \operatorname{Diff} / S^{1}$ of $\varphi \in$ Diff

$$
s([\varphi])=\left(\varphi_{0}, 0\right) \in \mathrm{Vir},
$$

where $\varphi_{0}(t)=\varphi\left(t+\varphi^{-1}(0)\right)$ is the element of $[\varphi]$ that satisfies $\varphi_{0}(0)=0$. If $u: \operatorname{Diff} / S^{1} \rightarrow \mathfrak{c}$ is smooth, $s e^{u}$ is also a smooth section, holomorphic if (using (2.2)) $0=\bar{\partial}\left(s e^{u}\right)=s(\bar{\partial} u) e^{u}+\bar{\partial} s e^{u}$, i.e.,

$$
\bar{\partial} u=-s^{-1} \bar{\partial} s .
$$

Assuming we can prove (4.3) has a smooth, or only $C^{1}$ solution, Theorem 4.2 (and Theorem 1.1) is also proved. Below we will solve (4.3) in two different ways.

We start by computing the right hand side of (4.3). Since every class $[\varphi] \in \operatorname{Diff} / S^{1}$ contains a $\varphi_{0}$ that fixes $0 \in S^{1}$, we can identify Diff $/ S^{1}$ with Diff, the group of such diffeomorphisms; $T_{[\mathrm{id}]} \mathrm{Diff} / S^{1}$ with $T_{\mathrm{id}} \operatorname{Diff}_{0}=$ $\operatorname{diff}_{0}$, vector fields $v$ on $S^{1}$ that vanish in 0 ; and $T_{[\text {id] }}^{1,0} \operatorname{Diff} / S^{1}$ with $\operatorname{diff}{ }_{0}^{1,0}=$ $\left\{\sum_{0}^{\infty} a_{n} e^{i n t} d / d t \in \mathbb{C} \otimes \operatorname{diff}_{0}\right\}$. Given $\varphi \in \operatorname{Diff}_{0}$ and $v \in \mathbb{C} \otimes \operatorname{diff}_{0}$, let $L_{\varphi} v$ denote the left translate of $v$ by $\varphi$, and introduce

$$
\Gamma\left(\varphi, V \frac{d}{d t}\right)=\int_{0}^{2 \pi} \log \varphi^{\prime} d V^{\prime}, \quad \varphi \in \operatorname{Diff}_{0}, V \frac{d}{d t} \in \mathbb{C} \otimes \operatorname{diff}_{0}
$$


We find $s^{-1} d s\left(L_{\varphi} v\right)=(v,-\Gamma(\varphi, v))$ and for $v \in \operatorname{diff}_{0}^{0,1}$

$$
s^{-1} \bar{\partial} s\left(L_{\varphi} v\right)=-\frac{(1, \tau)}{2 i \operatorname{Im} \tau} \Gamma(\varphi, v) \in \mathbb{C} \otimes \mathfrak{c} \subset \mathbb{C} \otimes \text { vir. }
$$

Thus, solving (4.3) amounts to finding a function $\chi:$ Diff $_{0} \rightarrow \mathbb{C}$ satisfying $\bar{\partial} \chi\left(L_{\varphi} v\right)=\Gamma(\varphi, v)$ or

$$
\bar{\partial} \chi=\gamma
$$

where $\gamma$ is a $(0,1)$-form given by $\gamma\left(L_{\varphi} v\right)=\Gamma(\varphi, v)$ for $v \in \operatorname{diff}_{0}^{0,1}$. (With the identification $\mathfrak{c} \approx T_{1} \mathbb{C}^{*}=\mathbb{C}, \chi=\bar{\tau} u$.) An alternative expression for $\gamma$ in the trivialization of $\mathbb{C} \otimes T$ Diff $_{0}$ as in section 3 is

$$
\gamma(\varphi, \dot{\varphi})=\int_{0}^{2 \pi} \log \varphi^{\prime} d\left(\dot{\varphi} / \varphi^{\prime}\right), \quad(\varphi, \dot{\varphi}) \in T_{\varphi}^{0,1} \operatorname{Diff}_{0}
$$

It is plain that $s^{-1} \bar{\partial} s$, hence $\gamma$, is closed: $\bar{\partial} \gamma=0$.

First solution of (4.4). We will make use of a general existence theorem for the $\bar{\partial}$ equation due to Raboin, see [21]. Thus, let $H$ be a complex Hilbert space, $Q: H \rightarrow H$ a self adjoint operator such that $Q^{1 / 3}$ is HilbertSchmidt, and $\Omega \subset H$ a pseudoconvex open set. (Pseudoconvexity means the existence of a plurisubharmonic function $\sigma: \Omega \rightarrow \mathbb{R}$ whose level sets $\{\sigma<a\}$ are at positive distance to $\partial \Omega, a \in \mathbb{R}$. For the equivalence of this with other notions of pseudoconvexity, see, e.g., [8].) Let $\beta$ be a closed $(0,1)$-form of class $C^{1}$ and bounded type on $\Omega$. This latter means that $\beta(v)\left(v \in T_{z} \Omega\right)$ remains bounded if $z$ is restricted to a bounded subset of $\Omega$ at positive distance to $\partial \Omega$, and $\|v\| \leq 1$. Raboin's theorem asserts that under these assumptions there is a $C^{1}$ function $\alpha$ on $\Omega \cap Q H$ such that $\bar{\partial} \alpha=\left.\beta\right|_{\Omega \cap Q H}$. As $\Omega \cap Q H$ is not an open subset of $H$, this needs some clarification. By $\alpha$ being $C^{1}$ we mean it is $C^{1}$ with respect to the smooth structure of $\Omega \cap Q H$ induced by $Q$, i.e., $\alpha \circ Q$ is $C^{1}$ on the open set $Q^{-1}(\Omega) \subset H$. (Strictly speaking, Theorem 2 of [21] assumes $\beta$ is $C^{\infty}$, but the proof needs only $C^{1}$. Nor is the other assumption that $\Omega$ is bounded essential, as explained there.)

We will apply this result as follows. Recall that Diff 0 Diff $/ S^{1}$ is biholomorphic to $\mathcal{S}=\left\{f(\zeta)=\zeta+a_{2} \zeta^{2}+\cdots \in \mathcal{F}\right\}$ by [9]. Instead of Diff $/ S^{1}$ we will work on spaces of holomorphic functions. With a given 
integer $r \geq 2$, let $A_{1}^{r}(\Delta)$ denote the set of holomorphic functions $f: \Delta \rightarrow \mathbb{C}$, $f(\zeta)=\zeta+\sum_{1}^{\infty} a_{n} \zeta^{n}$ that satisfy

$$
\|f\|=\|f\|_{r}=\left(\sum_{1}^{\infty} n^{2 r}\left|a_{n}\right|^{2}\right)^{1 / 2}<\infty .
$$

If the sum of $f(\zeta)=\zeta+f_{0}(\zeta)$ and $g(\zeta)=\zeta+g_{0}(\zeta)$ is defined $\zeta+f_{0}(\zeta)+g_{0}(\zeta)$, and multiplication of $f$ by $\mu \in \mathbb{C}$ is defined as $\zeta+\mu f_{0}, A_{1}^{r}(\Delta)$ becomes a Hilbert space. We claim that the open set

$$
\mathcal{S}^{r}=\left\{f \in A_{1}^{r}(\Delta): f \text { is embedding on } \bar{\Delta}\right\}
$$

is pseudoconvex.

Indeed, the continuous function $\sigma_{0}: \mathcal{S}^{r} \rightarrow \mathbb{R}$

$$
\sigma_{0}(f)=\sup \left\{\left|\frac{\zeta-\omega}{f(\zeta)-f(\omega)}\right|: \zeta, \omega \in \Delta, \zeta \neq \omega\right\}
$$

as the least upper bound of moduli of holomorphic functions, is plurisubharmonic, hence so is $\sigma(f)=\sigma_{0}(f)+\|f\|$. Also, it is straightforward to check that the sets $\{\sigma<a\}$ are at positive distance to $\partial \mathcal{S}^{r}$.

Next we pull back the form $\gamma$ to $\mathcal{S}$, a dense subset of $\mathcal{S}^{r}$, and realize it extends to a form of class $C^{1}$ on $\mathcal{S}^{r}$, provided $r \geq 6$. Indeed, given $f \in \mathcal{S}$, the corresponding $\varphi \in \operatorname{Diff}_{0}$ is obtained by considering the univalent representation $g$ of $\mathbb{C} \backslash \bar{\Delta}$ on the exterior of $f(\partial \Delta)$, such that $f(1)=g(1)$, and putting $\Phi=f^{-1} \circ g, \Phi\left(e^{i t}\right)=e^{i \varphi(t)}$. This construction also works if $f \in \mathcal{S}^{r}$ only, and gives a diffeomorphism $\varphi$ of somewhat less regularity; for example $\varphi \in \operatorname{Diff}_{0}^{r-2}$, i.e., $r-2$ continuous derivatives, are easy to prove. Furthermore, this $\varphi$ depends continuously on $f$.

More generally, with $k$ a nonnegative integer, the mapping

$$
\mathcal{S}^{r} \ni f \mapsto \varphi \in \operatorname{Diff}_{0}^{r-2-k}
$$

is of class $C^{k}$. Thus, if $k=2$, the differential of (4.7) will be $C^{1}$. Using this and (4.5), it is easy to check that the pullback of $\gamma$ by (4.7) extends to a $C^{1}$-form $\beta$ on $\mathcal{S}^{r}$, simply because the formula that defines this pullback makes sense on $\mathcal{S}^{r}$, provided $r \geq 6$. As $\mathcal{S} \subset \mathcal{S}^{r}$ is dense, $\beta$ is a closed $(0,1)$-form. 
Finally we show that when $r \geq 7, \beta$ is of bounded type. Let $\epsilon>0$ and

$$
F=\left\{f \in \mathcal{S}^{r}: \operatorname{dist}_{r}\left(f, \partial \mathcal{S}^{r}\right) \geq \epsilon,\|f\|_{r} \leq 1 / \epsilon\right\}
$$

where dist $_{r}$ refers to distance in $A_{1}^{r}(\Delta)$. Since the embedding $\mathcal{S}^{r} \rightarrow \mathcal{S}^{r-1}$ is compact, and $F$ is closed both in $\mathcal{S}^{r}$ and $\mathcal{S}^{r-1}$, we get that $F$ is in fact compact in $\mathcal{S}^{r-1}$. Similarly, the unit ball bundle in $\left.T \mathcal{S}^{r}\right|_{F}$ is compact in $T \mathcal{S}^{r-1}$. Since $\beta$ is continuous on the latter, it is bounded on the former, i.e., $\beta$ is of bounded type.

Define a Hilbert-Schmidt operator $R$ on $A_{1}^{r}(\Delta)$ by $R\left(\zeta+\sum_{1}^{\infty} a_{n} \zeta^{n}\right)=$ $\zeta+\sum_{1}^{\infty} a_{n} \zeta^{n} / n$. Then the range of $Q=R^{3}$ will contain $\mathcal{S}$, so by Raboin's theorem there is a $C^{1}$ function $\alpha$ on $\mathcal{S}$ that solves $\bar{\partial} \alpha=\left.\beta\right|_{\mathcal{S}}$. Transporting $\alpha$ back to Diff 0 we obtain a solution $\chi$ of (4.4).

Second solution of (4.4). This one is more algebraic, and exhibits a rather explicit solution. Along with $\gamma$ consider the one-form $\widetilde{\gamma}$ given by $\widetilde{\gamma}\left(L_{\varphi} v\right)=$ $\Gamma(\varphi, v)$ for $v \in \mathbb{C} \otimes \operatorname{diff}_{0}$. Thus $\gamma$ is the $(0,1)$ component of $\widetilde{\gamma}$. Straightforward application of Cartan's formula identifies $d \widetilde{\gamma}=\omega$ as the left invariant $(1,1)$-form whose value at id $\in$ Diff $_{0}$ is given by the Gelfand-Fuks cocycle $c$ (more exactly, the restriction of $c$ to the Lie algebra $\left.\mathbb{C} \otimes \operatorname{diff}_{0}\right)$. Hence a solution $\chi$ of (4.4) will also solve $\partial \bar{\partial} \chi=\omega$.

Now some solution $\chi_{0}$ of this latter equation can be constructed by embedding the space Diff $0 \approx$ Diff $/ S^{1}$ into a Grassmannian, and explicitly solving the $\partial \bar{\partial}$ equation there in terms of infinite determinants. Kirillov and Yuriev do this in [11] with a Lagrangian Grassmannian; the embedding is obtained from a symplectic representation of Diff. Alternatively, the unitary representations considered in section 3 can also be used; below we will sketch this approach.

Thus, fix $\lambda \in \mathbb{C}, \operatorname{Re} \lambda=\frac{1}{2}$. With $U_{\varphi}$ the representation of Diff on $L^{2}\left(S^{1}\right)=H=H_{+} \oplus H_{-}$as in section 3 , and $\Xi(\varphi) \in L^{2}\left(H_{+}, H_{-}\right)$the local coordinate of $U_{\varphi}\left(H_{+}\right) \in \operatorname{Gr}^{\star}\left(\varphi \in \operatorname{Diff}_{0}\right)$ given by (3.11), put

$$
D(\varphi)=D_{\lambda}(\varphi)=\operatorname{det}\left(I+\Xi^{*}(\varphi) \Xi(\varphi)\right) .
$$

If $\psi \in \operatorname{Diff}_{0}, U_{\psi}$ is in the restricted unitary group (see [20]), and we can decompose it according to the polarization $H=H_{+} \oplus H_{-}$as

$$
U_{\psi}=\left(\begin{array}{cc}
p & q \\
r & s
\end{array}\right)
$$


As proved in section $3, p$ and similarly $s$ are invertible, and $q, r$ are HilbertSchmidt. One easily verifies

$$
D(\psi \varphi)=D(\varphi) \operatorname{det} p^{*} p \mid \operatorname{det}\left(I+\left.p^{-1} q \Xi(\varphi)\right|^{2} .\right.
$$

Since $\Xi$ is holomorphic on Diff, it follows that $\partial \bar{\partial} \log D$ is left invariant. Therefore, to identify this (1,1)-form, it suffices to compute it at $\varphi=$ id. In this point $\partial \bar{\partial} \log D=\operatorname{tr} \partial \bar{\partial} \Xi^{*} \Xi=-\operatorname{tr} \bar{\partial} \Xi^{*} \wedge \partial \Xi=-\operatorname{tr} d \Xi^{*} \wedge d \Xi$. This latter can be computed using (3.12) that expresses the differential of $-\Xi$ at id, and we find that with $v=\left(e^{i n t}-1\right) d / d t \in \operatorname{diff}_{0}^{1,0}, w=\left(e^{-i m t}-1\right) d / d t \in \operatorname{diff}_{0}^{0,1}$ (thus $n, m>0$ )

$$
\left(-\operatorname{tr} d \Xi^{*} \wedge d \Xi, v \wedge w\right)= \begin{cases}|\lambda|^{2} n^{3}-\left(n^{3}-n\right) / 6 & \text { if } m=n \\ 0 & \text { if } m \neq n\end{cases}
$$

Since

$$
(\omega, v \wedge w)= \begin{cases}-2 i n^{3} & \text { if } m=n \\ 0 & \text { if } m \neq n\end{cases}
$$

the function

$$
\chi_{0}=-\frac{2 i}{|\lambda|^{2}-|\mu|^{2}} \log \frac{D_{\lambda}}{D_{\mu}}
$$

with $|\lambda| \neq|\mu|$ will satisfy $\partial \bar{\partial} \chi_{0}=\omega$ at id, hence everywhere by invariance.

With this $\chi_{0}$ we have $d\left(\tilde{\gamma}+\partial \chi_{0}\right)=d \tilde{\gamma}-\partial \bar{\partial} \chi_{0}=0$. Diff $f_{0}$ being contractible, there is a function $\chi$ (obtained from $\tilde{\gamma}+\partial \chi_{0}$ by a simple integration) that satisfies $d \chi=\tilde{\gamma}+\partial \chi_{0}$. Taking $(0,1)$ parts we conclude that $\chi$ solves (4.4).

\section{Acknowledgement}

Part of this research was done while the author enjoyed the hospitality of Eötvös University, Budapest.

\section{References}

1. V. Aldaya and J. Navarro-Salas, Quantization on the Virasoro group, Commun. Math. Phys. 126 (1990), 575-595.

2. R. Bott, On the characteristic classes of groups of diffeomorphisms, Ens. Math 23 (1977), 209-220.

3. M. J. Bowick and S. G. Rajeev, String theory as the Kähler geometry of loop space, Phys. Rev. Lett. 58 (1987), 535-538.

4. - The holomorphic geometry of closed bosonic string theory and Diff $S^{1} / S^{1}$, Nucl. Phys. B293 (1987), 348-384. 
5. I. M. Gelfand and D. B. Fuks, Cohomology of the Lie algebra of vector fields on the circle, Funk. Anal. Pril. 2 (1968), 92-93.

6. R. Goodman and N. R. Wallach, Projective unitary positive energy representations of $\operatorname{Diff}\left(S^{1}\right)$, J. Funct. Anal. 63 (1985), 299-321.

7. R. Hamilton, The inverse function theorem of Nash and Moser, Bull. Amer. Math. Soc. 7 (1982), 65-222.

8. A. Hirschowitz, Prolongement analytique en dimension infinie, Ann. Inst. Fourier 20 (1972), 255-292.

9. A. A. Kirillov, Kähler structures on K-orbits of the group of diffeomorphisms of a circle, Funk. Anal. Pril. 21(2) (1987), 42-45.

10. A. A. Kirillov and D. V. Yuriev, Kähler geometry of the infinite dimensional homogeneous space $M=$ Diff $_{+} S^{1} / S^{1}$, Funk. Anal. Pril. 21(4) (1987), 35-46.

11. _ Representation of the Virasoro algebra by the orbit method, J. Geom. Phys. 5 (1988), 351-363.

12. H. La, P. Nelson and A. S. Schwarz, Virasoro model space, Commun. Math. Phys. 134 (1990), 539-554.

13. C. Lebrun, A Kähler structure on the space of string world sheets, Class. Quant. Gravity 10 (1993), 141-148.

14. L. Lempert, Loop spaces as complex manifolds, J. Differential Geom. 38 (1993), 519-543.

15. J. Mickelsson, String quantization on group manifolds and the holomorphic geometry of Diff $S^{1} / S^{1}$, Commun. Math. Phys. 112 (1987), 653-661.

16. J. W. Milnor, Remarks on infinite dimensional Lie groups, Relativity, groups, and topology II, Les Houches session (B. deWitt and R. Stora, eds.), vol. XL, 1983, North-Holland, Amsterdam, 1984.

17. Y. A. Neretin, Representations of Virasoro and affine Lie algebras, Representation Theory and Noncommutative Harmonic Analysis 1 (A. A. Kirillov, Encyclopedia of Math. Sci., ed.), vol. 22, Springer-Berlin-Heidelberg, 1994.

18. A. Newlander and L. Nirenberg, Complex analytic coordinates in almost complex manifolds, Ann. of Math. 65 (1957), 391-404.

19. A. Pfluger, Über die Konstruktion Riemannscher Flächen durch Verhäftung, J. Indian Math. Soc. 24 (1961), 401-412.

20. A. Pressley and G. Segal, Loop groups, Clarendon Press, Oxford, 1990.

21. P. Raboin, Le problème du $\bar{\partial}$ sur un espace de Hilbert, Bull. Soc. Math. France 107 (1979), 225-240.

22. M. A. Virasoro, Subsidiary conditions and ghosts in dual resonance models, Phys. Rev. D1 (1970), 2933-2936.

23. E. Witten, Coadjoint orbits of the Virasoro group, Commun. Math. Phys. 114 (1988), 1-53.

P urdue University, West Lafayette, in 47907

E-mail address: lempert@math.purdue.edu 